The cases in which the Jacobian determinant of $T$ has at least one non-zero element, say $f_{u}(0,0) \neq 0$, are completely discussed. Certain cases where all $f_{v}, f_{v}, \phi_{u}, \phi_{v}$ are zero when $u=0, v=0$ are treated. If $f$ and $\phi$ admit a common factor in $R$, then there is an explosive point in $\bar{R}$, having an infinitely many valued inverse. Even then $\bar{R}$ may be the complete neighborhood of this point, the number of branches which are continuous outside this point being different in different subregions of $\bar{R}$.

53. It is well known that the group of isomorphisms of a group of order $p$ is of order $p-1$, and that of a cyclic group of order $p^{2}$ is of order $p(p-1)$. The corresponding group of the non-cyclic group of order $p^{2}$ is simply isomorphic with the linear homogeneous group on $p^{2}$ variables.

The groups of isomorphisms of all types of groups of order $p^{3}$ are determined by Western in his paper on "Groups of order $p^{3} q$," Proceedings of the London Mathematical Society, volume 30 .

Professor Marriott has determined the groups of isomorphisms of all types of groups of order $p^{4}$. He exhibits these as substitution groups and determines the order of each.

F. N. Core, Secretary.

\title{
ON 'THE NEGATIVE DISCRIMINANTS FOR WHICH THERE IS A SINGLE CLASS OF POSITIVE PRIMITIVE BINARY QUADRATIC FORMS.
}

BY PROFESSOR L. E. DICKSON.

(Read before the American Mathematical Society, April 29, 1911.)

For such a discriminant $-P$, the problem of the representation of numbers by a binary quadratic form of discriminant $-P$ is quite elementary; moreover, factorization into primes is unique in a quadratic field of discriminant $-P$. The only*

* E. Landau, Mathematische Annalen, vol. 56 (1903), p. 671. His method is not applicable to discriminants $-P$, where $P$ is odd, as was pointed out by M. Lerch, ibid., vol. 57 (1903), p. 568 . Results obtained by the latter by use of a relation between numbers of classes will here be proved by more elementary means and extensions given. 
such discriminants of the form $-4 k$ are those having $k=1,2$, $3,4,7$, as was conjectured by Gauss * after an examination of the determinants as far as -3000 . The present note gives practical criteria and the result of an examination of the values of $P$ less than one and one half million. We denote $a x^{2}+b x y+c y^{2}$ by $(a, b, c)$ and call $b^{2}-4 a c$ its discriminant.

First, let $P \equiv 0(\bmod 4)$. Then $(1,0, P / 4)$ must be the only reduced primitive form of discriminant $-P$. The case in which $P / 4$ is divisible by two distinct primes is excluded, since we may then express $P / 4$ as the product of two relatively prime factors $a$, $c$, such that $1<a<c$, and hence obtain the new primitive reduced form $(a, 0, c)$ of discriminant $-P$. Hence $P=4 p^{e}$, where $p$ is a prime. For $p=2,\left(4,4,2^{e-2}+1\right)$ is a primitive reduced form of discriminant $-P$ if $e \geqq 4$, and $(3,2,3)$ is one if $e=3$; while for $e=1$ or 2 , whence $P=8$ or 16, there is a single primitive reduced form. Next, let $p>2$. The even number $p^{e}+1$ cannot have an odd factor $>1$, since otherwise it would equal the product of two relatively prime integers $a$ and $c$, such that $1<a<c$, and $(a, 2, c)$ would give a new primitive reduced form of discriminant $-P$. Hence $p^{e}+1=2^{k}$. Then $\left(8,6,2^{k-3}+1\right)$ or $(5,4,7)$ is a primitive reduced form of discriminant $-P$ if $k>5$ or $k=5$, respectively. For $k=4,2^{k}-1=15$ is not a power of a prime. For $k=1,2,3, P=4,12,28$, there is a single primitive reduced form.

Next, let $P \equiv 3(\bmod 4)$. Then $\left[1,1, \frac{1}{4}(1+P)\right]$ must be the only reduced primitive form of discriminant $-P$. If $P=r s$, where $r$ and $s$ are relatively prime and $>1$, one of the factors is $\equiv 3(\bmod 4)$ and the other $\equiv 1(\bmod 4)$. Let $r>s$. Then $[(r+s) / 4,(r-s) / 2,(r+s) / 4]$ is a new primitive form of discriminant $-P$, which is reduced if $3 s \geqq r$. Its second right neighboring form (obtained by using $\delta=-1, \delta^{\prime}=0$ ) is $\left[s,-s, \frac{1}{4}(r+s)\right]$, which is reduced if $3 s<r$. Hence $P=p^{e}$, where $p$ is a prime $\equiv 3(\bmod 4)$ and $e$ is odd. If $p>3, e \geqq 3$, the form with $\alpha=\frac{1}{4}(p+1), b=1, c=\left(p^{e}+1\right) /(p+1)$ is a new primitive reduced form of discriminant $-P$; indeed, $c>4 a$ since $p^{e-1} \geqq p^{2}>p+2$. For $P=27,(1,1,7)$ is the only primitive reduced form. For $P=3^{e},\left[9,3, \frac{1}{4}\left(3^{e-2}+1\right)\right]$ or $(7,3,9)$ is a primitive reduced form if $e>5$ or $e=5$, respectively. Thus, if $P \neq 27, P$ must be a prime. Set

$$
T_{j}=\frac{1}{4}\left[(2 j+1)^{2}+P\right]=T_{0}+j(j+1) .
$$

* Disquisitiones Arithmeticae, Art. 303. 
If $j=q m+r, 0 \leqq r<m$, then $T_{j} \equiv T_{r} \equiv T_{m-r-1}(\bmod m)$. For $r>\frac{1}{2}(m-1), m-r-1<\frac{1}{2}(m-1)$. Hence any $T_{j}$ is congruent modulo $m$ to some $T_{r}$, where $0 \leqq r \leqq \frac{1}{2}(m-1)$. Let $2 g+1$ be the greatest odd integer $\leqq \sqrt{P / 3}$. In a reduced form $(a, b, c), b>0$, we have $b=2 \beta+1 \leqq 2 g+1, \beta \leqq g$. We shall prove that there is a single reduced form of discriminant $-P$ if and only if $T_{0}, T_{1}, \ldots, T_{g}$ are all prime numbers. It they are primes, a reduced form has $a=1, b=1$. Conversely, let there be a single reduced form. If $T_{0}$ were composite, there would be a reduced form with $b=1, a>1$. Suppose that $T_{0}, \ldots, T_{\beta-1}$ are primes, but $T_{\beta}=a c, c \geqq a>1$, where $0<\beta \leqq g$. If $a \geqq b$, where $b=2 \beta+1,(a, b, c)$ would be reduced. Hence $a<b$. Applying the above result for $m=a$, we see that $T_{\beta} \equiv T_{r}(\bmod a)$, where $r$ is some integer $0 \leqq r \leqq \frac{1}{2}(a-1)$. Thus $r<\beta$, so that $T_{r}$ is a prime. But $T_{r} \equiv T_{\beta} \equiv 0(\bmod a)$. Hence $T_{r}=a . \quad$ Thus $a \geqq T_{0} \geqq \frac{1}{4}(1+P)$. $P \geqq 3(2 g+1)^{2} \geqq 3(2 \beta+1)^{2}>3 a^{2}, a>\frac{1}{4}\left(1+3 a^{2}\right)$. Thus $(3 a-1)(a-1)<0$, which contradicts $a>1$.

If $P$ is a prime $<27$, then $g=0$ and the condition is that $T_{0}=\frac{1}{4}(1+P)$ shall be a prime. This is satisfied when $P=$ $3,7,11,19$.

For $P \equiv 7(\bmod 8), P>7, T$ is even and $>2$.

For $P \equiv 3(\bmod 8)$, set $P=8 k-5 . \quad$ For $k \equiv 2(\bmod 3)$, $k \geqq 5, T_{0}=2 k-1$ is divisible by 3 and exceeds 3 ; while for $k=2, P=11$. For $k \equiv 1(\bmod 3), P$ is divisible by 3 . For $k \equiv 0(\bmod 3), P=24 t-5$. For $t \equiv 1,4$, or $0(\bmod$ 5), $T_{0}=6 t-1, T_{1}=6 t+1$ or $P$ is divisible by 5 and exceeds 5 except when $t=1, P=19$. For $t=2$ or $3, P=43$ or 67 and $g=1$, while $T_{0}$ and $T_{1}$ are primes. For $t=7, P=163$, $g=3$, and $T_{0}, T_{1}, T_{2}, T_{3}$ are primes $41,43,47,53$. For $t=8$, $P=11.17$. There remain the cases $t=5 l+12,5 l+13$, where $l \geqq 0$. Hence we may state the

TheOREM. There is a single class of positive primitive quadratic forms of negative discriminant $-P$ when

$$
P=3,4,7,8,11,12,16,19,27,28,43,67,163 ;
$$

but more than one class if $P$ is not one of these 13 numbers and not a prime of the form $120 l+283$ or $120 l+307, l \geqq 0$.

The remaining primes $<1000$ are $P=283,523,643,883$, $307,547,787,907$. For these $g \geqq 4, T_{2}=77, T_{1}=7 \cdot 19$, $T_{0}=7 \cdot 23, \quad T_{0}=13.17, \quad T_{0}=77, T_{2}=11.13, T_{2}=7 \cdot 29$, 
$T_{4}=13.19$, respectively. Hence in each case there is more than one class.

A practical method of examining a wide range of values of $P$ consists in first excluding the values of $l$ for which any one of the numbers $T_{0}, \ldots, T_{g}$ has a given small prime factor $p$. For $P=120 l+283$ or $307, T_{0}=30 l+71$ or $30 l+77$, $g \geqq 4$. This exclusion has already been effected for $p=3$ or 5 . For $p=7$, any $T_{j}$ is congruent to $T_{0}, T_{1}, T_{2}$ or $T_{3}$. For $T_{0}=$ $30 l+77$, these are divisible by 7 if $l \equiv 0,6,4,1(\bmod 7)$, respectively; for $30 l+71$, if $l \equiv 3,2,0,4(\bmod 7)$. Hence there remain the cases

$$
T_{0}=210 m+\mu, \mu=137,167,227,101,221,251 .
$$

The least $P$ is now 403 , whence $d \geqq 5$. Now $T_{0} \equiv m+\mu$ $(\bmod 11)$. Thus $T_{0}$ is divisible by 11 if $m \equiv 6,9,4,9,10$, $2(\bmod 11)$, respectively. But $T_{l_{c}}=T_{k-1}+2 k$. Hence if we subtract $2 k$ from the $m$ for which $T_{k-1} \equiv 0(\bmod 11)$, we obtain the $m$ for which $T_{k} \equiv 0(\bmod 11)$. This may be done by counting spaces on square ruled paper. At each point so obtained a hole is punched, thus giving a $6 \times 11$ stencil for $p=11$. The least $T_{0}$ is now 221 , whence $P \geqq 883, g \geqq 8$. Similarly, stencils were constructed for $p=13,17,19,23,29$. After using the first three stencils, it was noted that $m \geqq 4$ for each $\mu$, whence $T_{0} \geqq 941, P \geqq 3763, g \geqq 17$.

The first 10710 values of $T_{0}$ were examined; to this end $m$ was given the values $\leqq 1785$. The use of each stencil excluded more than half of the values left at the earlier stage. After using the stencils for $p \leqq 29$, we had left 110 numbers, for each of which $T_{0}, \ldots$, or $T_{6}$ was verified to be composite. In just four cases were $T_{0}, \ldots, T_{5}$ all prime. The work, including the making of the stencils, was done in two days.

Theorem. For $163<P<1,500,000$ there is more than one class of positive primitive quadratic forms of discriminant $-P$.

For a greater $P, g \geqq 353$ and there is more than one class unless $T_{0}, T_{1}, \ldots, T_{353}$ are all primes. The chance that such a case will arise is extremely small. Note that, for $P$ not exceeding $1 \frac{1}{2}$ millions, $T_{0}, \cdots, T_{14}$ were shown to be not all prime. 